\title{
Characterization of Immunological Niches within Peyer's Patches by ex vivo Photoactivation and Flow Cytometry Analysis
}

Adi Biram and Ziv Shulman*

Department of Immunology, Weizmann Institute of Science, Rehovot, Israel

*For correspondence: ziv.shulman@weizmann.ac.il

[Abstract] T follicular helper (Tfh) cells regulate B cell selection for entry into the germinal center (GC) reaction or for differentiation into antibody forming cells. This process takes place at the border between the $\mathrm{T}$ and $\mathrm{B}$ zones in lymphoid organs and involves physical contacts between $\mathrm{T}$ and $\mathrm{B}$ cells. During these interactions, $T$ cells endow the $B$ cells with selection signals that promote $G C$ seeding or plasmablast differentiation based on their B cell receptor affinity. In Peyer's patches (PPs), T cells promote B cell colonization of the subepithelial dome (SED) without effective affinity-based clonal selection. To specifically characterize the $T$ cell population that resides within the SED niche, we performed ex vivo photoactivation of the SED compartment followed by flow cytometry analysis of the labeled cells, as described in this protocol. This technique integrates both spatial and cellular information in studies of immunological niches and can be adapted to various experimental systems.

Keywords: Photoactivation, Two-photon microscopy, SED, Tfh cells, Flow cytometry

[Background] Affinity maturation, the process wherein the affinity of serum antibodies towards a specific antigen increases over time, is achieved by selection of B cells bearing high affinity BCRs within germinal centers (GCs). Increase in antibody affinity is mediated through iterative cycles of somatic hyper mutation and affinity-based selection, a process which is orchestrated by T follicular helper (Tfh) cells (Kepler and Perelson, 1993; Oprea and Perelson, 1997; Victora and Nussenzweig, 2012). The GC is comprised of two microanatomical sites; the dark zone, where $B$ cells proliferate and acquire somatic hypermutations, and the light zone, where B cells interact with cognate antigen and T cells. Although intravital imaging techniques were able to define immune cell dynamics in GCs (Allen et al., 2007; Hauser et al., 2007; Schwickert et al., 2007), transition between the GC zones remained in question. This problem was later solved by the generation of mice expressing photoactivatable GFP (PA-GFP) (Victora et al., 2010).

PA-GFP is a GFP variant whose peak excitation wavelength shifts from $415 \mathrm{~nm}$ (inactive PA-GFP) to $495 \mathrm{~nm}$ (active PA-GFP) upon two-photon irradiation at $830 \mathrm{~nm}$. The non-activated PA-GFP is fluorescent as well, and this property can be used to distinguish between the photoactivated area and the total cells (Patterson and Lippincott-Schwartz, 2002).

The use of mice expressing PA-GFP provided direct evidence for interzonal migration in the GC and for the definition of the major GC exit zone (Victora et al., 2010; Stoler-Barak et al., 2019). Combination of intravital two-photon laser scanning microscopy with in situ photoactivation, allowed the 
microanatomical labeling of distinct niches within the germinal center, and led to the discovery that $T$ cell help controls the movement between the two GC zones (Victora et al., 2010).

Cell-surface markers are commonly used to define a cell population in a specific niche; however, distinctive markers are not always available, generating a gap between the cellular and spatial information in the studied tissue. Furthermore, unknown cell populations that reside within a specific niche are usually hard to detect and characterize by conventional techniques. To overcome these limitations, photoactivation-based approaches have been used for unbiased identification of tissueresident immune cells with minimal a priori knowledge of unique cell-surface marker expression. Nichespecific landmarks are often introduced into mice prior to labeling by photoactivation to define the area of interest. For example, adoptive cell transfer of fluorescently labeled B cells mark the B cell area within a tissue and can guide the selection of the region of interest for photoactivation (Medaglia et al., 2017). In the study associated with this protocol and as described here, we specifically photoactivated the subepithelial dome niche within the Peyer's patch (Biram et al., 2019). This protocol can be adapted to other niches and additional tissues of interest.

\section{Materials and Reagents}

1. Sterile Syringe $3 \mathrm{ml}$, luer lock (MedHarmony, catalog number: 181110)

2. Cell Strainer Nylon, Frame PP, pore size $70 \mu \mathrm{m}$, sterile (SPL Life Sciences, catalog number: 93070)

3. Monoject ${ }^{\mathrm{TM}} 18 \mathrm{G}$ blunted cannula (Covidien, catalog number: 8881202348 )

4. High precision microscope cover glasses $18 \times 18 \mathrm{~mm}, 1.5 \mathrm{H}$ (Marienfeld, catalog number: 0107032)

5. Sandblasted single frosted pre-cleaned microscope slides, $25 \times 75 \mathrm{~mm} \times 1 \mathrm{~mm}$ thick (Thermo Fisher Scientific, catalog number: 421-004T)

6. UBC PA-GFP mouse (The Jackson Laboratory, catalog number: 022486)

This mouse strain carries a transgene of a photoactivatable variant of the GFP protein under the regulatory control of the constitutively expressed human ubiquitin $C$ (UBC) promoter. Therefore, it enables rapid and stable fluorescent labeling of living cells.

7. Calcium and Magnesium free phosphate buffered saline (PBS-/-) (Biological Industries, catalog number: 02-023-1A)

8. Ethylenediaminetetraacetic acid solution (EDTA) (Sigma-Aldrich, catalog number: 03690)

9. Fetal Bovine Serum, charcoal stripped (Thermo Fisher Scientific, catalog number: 12676029)

10. Silicone grease (can be found in hardware stores)

11. Double distilled water (DDW)

12. TruStain FcX'TM (anti-mouse CD16/32) Antibody (Biolegend, clone: 93, catalog number: 101319)

13. Brilliant violet 605 anti-mouse B220 (CD45R) antibody (Biolegend, clone: RA3-6B2, catalog number: 103243) 
14. APC-Alexa Fluor 750 anti-mouse CD4 antibody (Thermo Fisher Scientific, clone:S3.5, catalog number: MHCD0427)

15. PE anti-mouse CD44 antibody (Biolegend, clone: IM7, catalog number: 103023)

16. Alexa Fluor 700 anti-mouse CD62L antibody (Thermo Fisher Scientific, clone: MEL-14, catalog number: 56-0621-82)

17. PE/Cy7 anti-mouse CD279 (PD-1) antibody (Biolegend, clone: RMP1-30, catalog number: 109109)

18. Biotin anti-mouse CD185 (CXCR5) antibody (Biolegend, clone: L138D7, catalog number: 145509)

19. Alexa Fluor 647 Streptavidin (Biolegend, catalog number: 405237)

20. FACS buffer (see Recipes)

\section{Equipment}

1. Tunable two-photon laser scanning microscope (TPLSM) equipped with a 20x water lens (Zeiss LSM 880 upright microscope fitted with Coherent Chameleon Vision laser)

2. CytoFlex flow cytometer (Beckman Coulter)

\section{Procedure}

Note: Photoactivation is a time consuming process, therefore it is recommended to evaluate the time necessary for photoactivation of all required niches and analyze a sample size where samples will not wait for more than $3 \mathrm{~h}$ for the following staining step. Longer delay in tissue processing might increase the frequency of dead cells and therefore will not contribute to pool of analyzed cells. In the case of the SED photoactivation, 30 SED regions were photoactivated in each mouse and up to two mice were analyzed a day. In addition, photoactivation can be held on the full PA-GFP mouse, on transferred cells or in chimeras prepared with PA-GFP bone marrow. Heterozygous PA-GFP mice can also be used in this protocol, but homozygous PA-GFP mice generate a stronger GFP signal. In the study associated with this protocol, we generated chimeric mice composed of $90 \%$ homozygous PA-GFP bone marrow and 10\% AID-GFP bone marrow. AID is expressed by SED and GC $B$ cells, thus AID-GFP was used as a landmark for these compartment.

1. Euthanize a mouse by cervical dislocation or by $\mathrm{CO}_{2}$ inhalation (the technique does not affect the following steps).

2. Open the abdominal cavity and remove the small intestine from the cecum to stomach into a PBS containing petri dish ( $15 \mathrm{ml}$ of PBS).

3. Flush the intestine with $40 \mathrm{ml}$ PBS using a $30 \mathrm{ml}$ syringe with an $18 \mathrm{G} \mathrm{Monoject}^{\mathrm{TM}}$ blunted cannula. Avoid contact of the tissue with the intestinal content flushed out. 
4. Excise a piece of the intestine that includes a Peyer's patch and cut it longitudinally without damaging the PP.

5. Place the tissue on a cover slip glass and add silicone grease around the tissue. Make sure that the silicone layer is not thicker than the tissue.

6. Add PBS $(\sim 30 \mu \mathrm{l})$ to hydrate the tissue and cover with an additional cover slip glass (Figure 1).
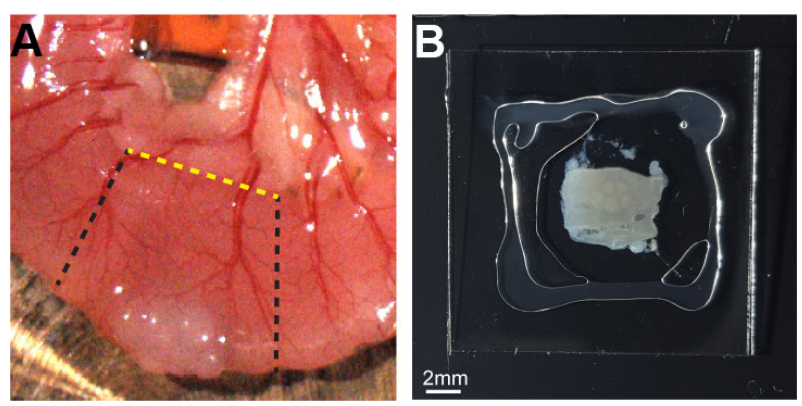

Figure 1. Peyer's patch slide preparation. A. Intestinal tissue containing a Peyer's patch was cut out (black dashed lines) and an additional longitudinal cut (yellow dashed line) was performed to expose the SED compartment. B. The PP was placed on a cover slip, and hydrated with PBS bound by silicone grease.

7. Place the tissue on a slide with the desired side for photoactivation facing up.

8. Add a drop of DDW on the tissue and place the lens in the region of interest.

9. Focus and image the tissue at $940 \mathrm{~nm}$ wavelength (this will also ensure that unspecific photoactivation will take place).

10. Define a $5 \mu \mathrm{m}$ interval Z-stack within the tissue in the area of desired photoactivation.

11. Image a high quality Z-stack image before photoactivation at $940 \mathrm{~nm}$ (Figure 2).

12. Define the area of photoactivation by adding a region in the ZEN setup and make sure to mark this region for acquisition.

13. Change laser parameter to $830 \mathrm{~nm}$ and image. This should include imaging of a specific region of interest with a Z-stack at $830 \mathrm{~nm}$.

14. Return laser to $940 \mathrm{~nm}$ and remove the marked region.

15. Repeat image acquisition at $940 \mathrm{~nm}$ (Figure 2). This should include the entire field with a Zstack. The photoactivated area will appear green according to the defined region boundaries. 


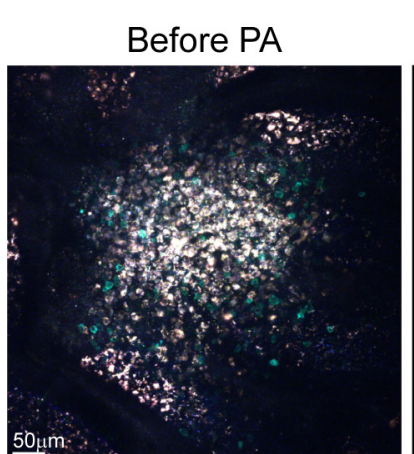

$940 \mathrm{~nm}$
During PA

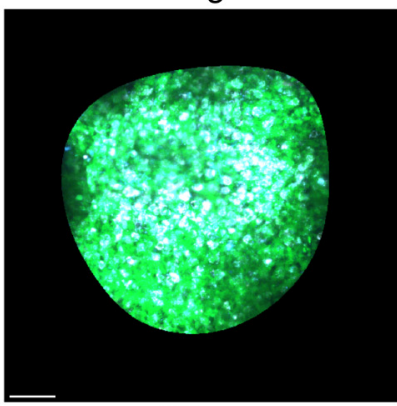

$830 \mathrm{~nm}$
After PA

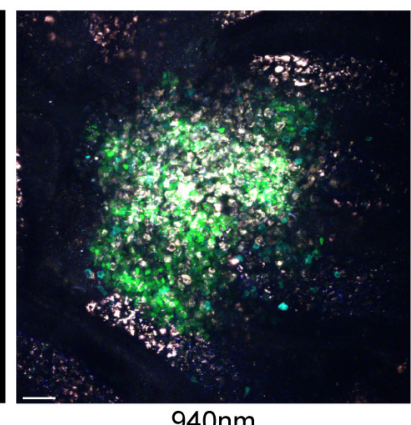

$940 \mathrm{~nm}$

Figure 2. Photoactivation of the SED area in a PP of a chimeric PA-GFP mouse. Images show the SED before photoactivation, during photoactivation at $830 \mathrm{~nm}$ and after photoactivation to indicate the labeled population.

16. Repeat for all desired niches.

17. Open cover slip glasses and gently transfer the tissue into a petri dish with a $70 \mu \mathrm{m}$ cell strainer and $3 \mathrm{ml} \mathrm{FACS}$ buffer.

18. Using a piston of a $3 \mathrm{ml}$ syringe mash the PPs to create a single-cell suspension of PP-derived cells.

19. Transfer the cell suspension into a FACS tube with a cell-strainer cap.

20. Wash the petri dish with additional $1 \mathrm{ml}$ of FACS buffer and collect the solution into the same FACS tube to maximize cell collection. The sample contains all PP cells, ranging 3-10 M cells.

21. Spin down the cells at $300 \mathrm{RCF}$ for $7 \mathrm{~min}$ at $4{ }^{\circ} \mathrm{C}$.

22. Discard the supernatant and resuspend the cells with the residual buffer (approximately $100 \mu \mathrm{l}$ ).

23. Add $1 \mu \mathrm{Fc}$ blocker antibody (TruStain $\mathrm{FcX}^{\mathrm{TM}}$ ), vortex and place on ice.

24. Incubate 5 min on ice.

25. Add $100 \mu$ staining mix prepared in FACS buffer. In the paper associated with this protocol, Tfh cell markers were used to stain for SED-derived T cells (B220- CD4 ${ }^{+}$CD44 $4^{+}$CD62 $L^{-}$PD- $1^{+}$ $\mathrm{CXCR5^{+ }}$ ). In cases where a biotin-conjugated antibody is used, first stain the cells with the biotin-conjugated antibody together with other conjugated antibodies, and in a second step stain the cells with the streptavidin antibody. Since the residual volume in the tube following blocking is approximately $100 \mu \mathrm{l}$, the antibody mix is prepared as a $2 x$ concentration to achieve final dilution as indicated in Table 1. 
Table 1. List of antibodies used for T cell staining in photoactivated PPs

\begin{tabular}{llll}
\hline Marker & Final dilution & Stock concentration $[\mathrm{mg} / \mathrm{ml}]$ & $\mu \mathrm{g} / 100 \mu \mathrm{l} \mathrm{mix}(2 \mathrm{x})$ \\
\hline BV605 B220 & $1: 400$ & 0.2 & 0.1 \\
APC-AF750 CD4 & $1: 400$ & 0.2 & 0.1 \\
PE CD44 & $1: 400$ & 0.2 & 0.1 \\
AF700 CD62L & $1: 400$ & 0.5 & 0.25 \\
PEICy7 PD-1 & $1: 600$ & 0.2 & 0.067 \\
Biotin CXCR5 & $1: 100$ & 0.5 & 1 \\
\hline
\end{tabular}

26. Vortex and incubate on ice for $30 \mathrm{~min}$ in the dark to allow surface marker staining.

27. Wash the cells with $2 \mathrm{ml} \mathrm{FACS}$ buffer and spin down the cells at $300 \mathrm{RCF}$ for 7 min at $4{ }^{\circ} \mathrm{C}$.

28. Add $100 \mu \mathrm{l}$ staining mix containing 1:400 AF647 Streptavidin antibody (stock concentration: $0.5 \mathrm{mg} / \mathrm{ml}$ ) prepared in FACS buffer.

29. Vortex and incubate on ice for $30 \mathrm{~min}$ in the dark to allow surface marker staining.

30. Wash the cells with $2 \mathrm{ml} \mathrm{FACS}$ buffer and spin down the cells at $300 \mathrm{RCF}$ for $7 \mathrm{~min}$ at $4{ }^{\circ} \mathrm{C}$.

31. Resuspend the cells with $500 \mu \mathrm{I}$ FACS buffer and analyze the cells using CytoFlex flow cytometer (Beckman Coulter).

\section{Notes}

1. Since the frequency of the photoactivated cell population is very low $(\sim 1-5 \%$ of total single live cells), record as many events as possible to achieve a reliable number of cells for further analysis.

2. In each experiment, extract and stain PPs derived from a mouse that was not photoactivated as a negative control. This control is crucial for proper gating on the photoactivated cell population. Gate on single live cells and out of this population gate on the $\mathrm{V} 00^{+} \mathrm{GFP}^{+}$cell population. The $\mathrm{V} 500^{+}$represent the entire photoactivatable cell population while the cells that are $\mathrm{GFP}^{+}$are the cells that were labeled by the two-photon microscope. This population can be analyzed for specific markers of interest. In the paper associated with this protocol we stained for Tfh cells as previously described (Liu, 2012).

\section{Recipes}

1. FACS buffer

Calcium and Magnesium free PBS (-/-)

$2 \%$ fetal bovine serum (FBS)

1 mM EDTA

To $500 \mathrm{ml}$ PBS add $10 \mathrm{ml}$ of PBS and $1 \mathrm{ml}$ of $0.5 \mathrm{M}$ EDTA

Note: FACS buffer is kept up to one month at $4{ }^{\circ} \mathrm{C}$. 


\section{Data analysis}

As previously described, in the work associated with this study (Biram et al., 2019), chimeric mice were used to label the SED and GC compartments. Full flow cytometry analysis of T cell populations is available in the original paper. As shown in the representative plots in Figure 3, photoactivated cells were gated out of single lymphocytes and analyzed according to the markers of interest. In the case of photoactivation of non-chimeric mice, such as the UBC-PA GFP mouse, two populations will appear on the plot: all cells will be $\mathrm{V} 500^{+}$and only the photoactivated cells will appear as $\mathrm{V} 500^{+}$ GFP $^{+}$. When photoactivating a sample of transferred cells, a V500- population will represent the endogenous cell population. Photoactivated cell frequency is relatively low ( 1-2\%) and for appropriate statistical analysis, it is recommended to record a total of $\sim 2 \mathrm{M}$ cells.

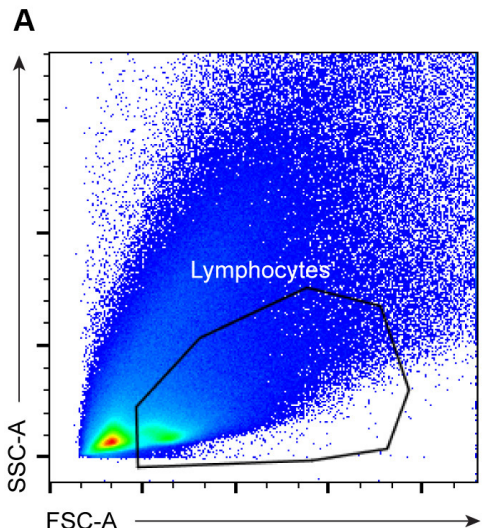

B

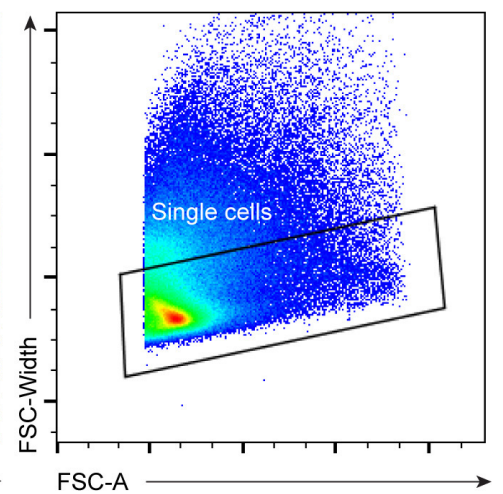

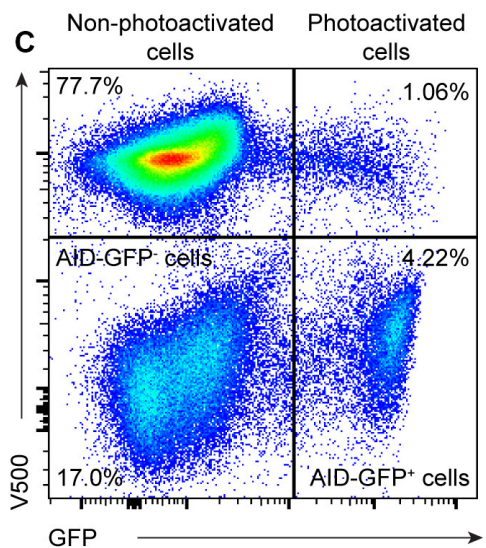

Figure 3. Gating strategy for photoactivated-cells in mouse PPs. Representative flow cytometry plots showing the gating strategy on the PA-GFP cell population in PA-GFP AID-GFP bone marrow chimeras as in Biram et al. (2019). A. Live lymphocytes were gated as shown. B. Doublets were removed using the FSA-A/FSC-width distribution. C. V500 was used to mark all PA-GFP derived cells. Non-photoactivated cells (V500+ GFP ${ }^{-}$), AID-GFP- cells, AID-GFP ${ }^{+}$cells, and photoactivated cells are represented as shown in the plot.

\section{Acknowledgments}

Z.S. is supported by the European Research Council (grant No. 677713), Human Frontiers of Science Program (grant No. CDA-00023/2016), Israel Science Foundation (grant No. 1090/18), Azrieli Foundation, Rising Tide Foundation and the Morris Kahn Institute for Human Immunology. Z.S. is a member in the European Molecular Biology Organization Young Investigator Program and is supported by grants from The Benoziyo Endowment Fund for the Advancement of Science, The Sir Charles Clore Research Prize, Comisaroff Family Trust, Irma \& Jacques Ber-Lehmsdorf Foundation, Gerald O. Mann Charitable Foundation and David M. Polen Charitable Trust.

This protocol provides details regarding photoactivation and flow cytometry analysis of Peyer's patch niches as previously described (Biram et al., 2019). 
Please cite this article as: Biram and Shulman, (2020). Characterization of Immunological Niches within Peyer's Patches by ex vivo Photoactivation and

\section{Competing interests}

The author declare no competing interests.

\section{Ethics}

All experimental procedures have been approved by the Weizmann Institute Animal Care and Use Committee (IACUC) and followed all relevant ethical regulations, IACUC number 00960118-4.

\section{$\underline{\text { References }}$}

1. Allen, C. D., Okada, T., Tang, H. L. and Cyster, J. G. (2007). Imaging of germinal center selection events during affinity maturation. Science 315(5811): 528-531.

2. Biram, A., Strömberg, A., Winter, E., Stoler-Barak, L., Salomon, R., Addadi, Y., Dahan, R., Yaari, G., Bemark, M. and Shulman, Z. (2019). BCR affinity differentially regulates colonization of the subepithelial dome and infiltration into germinal centers within Peyer's patches. Nat Immunol 20(4): 482-492.

3. Hauser, A. E., Junt, T., Mempel, T. R., Sneddon, M. W., Kleinstein, S. H., Henrickson, S. E., von Andrian, U. H., Shlomchik, M. J. and Haberman, A. M. (2007). Definition of germinal-center B cell migration in vivo reveals predominant intrazonal circulation patterns. Immunity 26(5): 655667.

4. Kepler, T. B. and Perelson, A. S. (1993). Cyclic re-entry of germinal center B cells and the efficiency of affinity maturation. Immunol Today 14(8): 412-415.

5. Liu, Z. (2012). FACS staining for follicular helper T cells. Bio-protocol 2(2): e35.

6. Medaglia, C., Giladi, A., Stoler-Barak, L., De Giovanni, M., Salame, T. M., Biram, A., David, E., Li, H., lannacone, M., Shulman, Z. and Amit, I. (2017). Spatial reconstruction of immune niches by combining photoactivatable reporters and scRNA-seq. Science 358(6370): 1622-1626.

7. Oprea, M. and Perelson, A. S. (1997). Somatic mutation leads to efficient affinity maturation when centrocytes recycle back to centroblasts. J Immunol 158(11): 5155-5162.

8. Patterson, G. H. and Lippincott-Schwartz, J. (2002). A photoactivatable GFP for selective photolabeling of proteins and cells. Science 297(5588): 1873-1877.

9. Schwickert, T. A., Lindquist, R. L., Shakhar, G., Livshits, G., Skokos, D., Kosco-Vilbois, M. H., Dustin, M. L. and Nussenzweig, M. C. (2007). In vivo imaging of germinal centres reveals a dynamic open structure. Nature 446(7131): 83-87.

10. Stoler-Barak, L., Biram, A., Davidzohn, N., Addadi, Y., Golani, O. and Shulman, Z. (2019). $\underline{B}$ cell dissemination patterns during the germinal center reaction revealed by whole-organ imaging. J Exp Med 216(11): 2515-2530.

11. Victora, G. D. and Nussenzweig, M. C. (2012). Germinal centers. Annu Rev Immunol 30: 429457. 
12. Victora, G. D., Schwickert, T. A., Fooksman, D. R., Kamphorst, A. O., Meyer-Hermann, M., Dustin, M. L. and Nussenzweig, M. C. (2010). Germinal center dynamics revealed by multiphoton microscopy with a photoactivatable fluorescent reporter. Cell 143(4): 592-605. 